Revista Arbitrada Interdisciplinaria KOINONIA

Año VI. Vol VI. N³. Edición Especial: Educación II. 2021

Hecho el depósito de Ley: FA2016000010 ISSN: 2542-3088

FUNDACIÓN KOINONIA (F.K). Santa Ana de Coro. Venezuela.

Isabel Fabiola Pauta-Ayabaca; Luis Bolívar Cabrera-Berrezueta

http://dx.doi.org/10.35381/r.k.v6i3.1340

\title{
Educación y COVID-19: Desafíos del docente de Bachillerato en tiempos de pandemia
}

\section{Education and COVID-19: Teacher's challenges in pandemic time}

\author{
Isabel Fabiola Pauta-Ayabaca \\ isabel.pauta.85@est.ucacue.edu.ec \\ Universidad Católica de Cuenca, Azogues \\ Ecuador \\ https://orcid.org/0000-0002-0326-623X \\ Luis Bolívar Cabrera-Berrezueta \\ bolivarcabrera@ucacue.edu.ec \\ Universidad Católica de Cuenca, Cuenca \\ Ecuador \\ https://orcid.org/0000-0002-6853-635X
}

Recepción: 10 de abril 2021

Revisado: 05 de mayo 2021

Aprobación: 30 de junio 2021

Publicación: 15 de julio 2021 
Revista Arbitrada Interdisciplinaria KOINONIA

Año VI. Vol VI. N`3. Edición Especial: Educación II. 2021

Hecho el depósito de Ley: FA2016000010 ISSN: 2542-3088

FUNDACIÓN KOINONIA (F.K). Santa Ana de Coro. Venezuela.

\title{
RESUMEN
}

El articulo tiene por objetivo determinar el papel y desafío del docente del Colegio de Bachillerato Ciudad de Cuenca, para enfrentar con éxito la enseñanza a los estudiantes en la crisis sanitaria. Metodológicamente de tipo descriptiva correlacional con cálculo de Chi-cuadrado de Pearson. Al relacionar estas dos variables se pudo determinar que el lugar donde el investigado utiliza para estudiar y las relaciones intrafamiliares no están asociadas, pues el coeficiente de Pearson en la prueba Chi-cuadrado es de 0,148 , valor mayor a 0,05 que asume $\mathrm{H} 0$ hipótesis nula, por tanto, no asociadas. Los docentes enfrentan desafíos que exigen de él, una mayor preparación, un profundo acercamiento al estudiante, una estrecha relación con el entorno familiar; exige, simplemente vocación, pasión y amor por lo que hace.

Descriptores: Método de enseñanza; enseñanza multimedia; publicación educacional. (Palabras tomadas del Tesauro UNESCO).

\begin{abstract}
The article aims to determine the role and challenge of the teacher of the Colegio de Bachillerato Ciudad de Cuenca, to successfully face the teaching of students in the health crisis. Methodologically descriptive correlational with Pearson's Chi-square calculation. By relating these two variables it was possible to determine that the place where the researcher uses to study and the intra-family relationships are not associated, since the Pearson coefficient in the Chi-square test is 0.148 , a value greater than 0.05 that $\mathrm{H} 0$ assumes hypothesis null, therefore, not associated. Teachers face challenges that require greater preparation, a deep approach to the student, a close relationship with the family environment; he simply demands vocation, passion and love for what he does.
\end{abstract}

Descriptors: Teaching methods; multimedia instruction; educational publications. (Words taken from the UNESCO Thesaurus). 


\section{INTRODUCCIÓN}

En la actualidad, el mundo soporta una de sus peores crisis a nivel económico y social, debido principalmente a la pandemia ocasionada por el virus SARS-CoV-2 que ha limitado la capacidad de reacción de las instituciones para enfrentar las consecuencias del virus, que ha socavado las estructuras económicas y sociales de la humanidad, que ha deteriorado y aumentado la brecha entre ricos y pobres, que ha desnudado la fragilidad de las políticas públicas de los estados para precautelar la seguridad de sus habitantes. Naturalmente, la educación no escapa de esta problemática, solo por citar un ejemplo más de 1500 millones de estudiantes han sufrido las consecuencias del cierre de escuelas, colegios y universidades a nivel mundial (Quevedo-Álava et al. 2020).

En nuestro país, los efectos han sido catastróficos, de acuerdo al Observatorio Social del Ecuador, afirma que 5 de cada 10 niños tienen acceso a la educación desde su casa a través del Internet, la mayoría de ellos asentados en zonas urbanas, la deserción escolar aumentó significativamente, depresión, ansiedad, agresividad se incrementó en la población escolar (Rodríguez-Tablado et al. 2020). Con este panorama, este artículo pretende analizar cuál debe ser el papel y el desafío del docente del Colegio de Bachillerato Ciudad de Cuenca, para enfrentar con éxito las problemáticas que significa la educación en tiempos de pandemia.

\section{Referencial teórico}

La educación se convierte en el instrumento más poderoso para cambiar la realidad del individuo y la sociedad, se convierte en el bastión para el desarrollo de los pueblos, mejora las condiciones de vida de la población y posibilita el crecimiento de la calidad de vida de los habitantes, por lo tanto, es "uno de los factores que afecta directamente el crecimiento y desarrollo de los individuos y sociedades, es la educación. La educación enriquece la cultura y creencias, y todo lo que caracteriza al ser humano (HurtadoTalavera, 2020). 
En este sentido, para lograr el desarrollo de la sociedad, los Estados tienen la obligación de mirar a la educación como uno de los pilares más importantes para el crecimiento de los pueblos, por tanto, los gobernantes deben en sus planes, programas y proyectos garantizar políticas públicas sostenibles en el tiempo para el fomento y desarrollo de la educación. Separando la crisis social y económica suscitada por la pandemia ocasionada por el Coronavirus, las actividades educativas durante la pandemia sufrieron un fuerte impacto por múltiples causas que obligaron a las autoridades educativas nacionales a tomar un conjunto de medidas que minimice ese impacto, medidas como ajustarse a un nuevo escenario formativo: la virtualidad (García-Cedeño et al. 2020).

El haber trasladado la estructura educativa presencial a la virtualidad significó una gran cantidad de riesgos, entre ellos, deserción escolar, hacinamiento, incremento de la violencia intrafamiliar hacia el escolarizado, procesos educativos aislados, sobre carga de tareas a los docentes, entre otros; todos ellos de alguna forma abonaron a que el aprendizaje, elemento clave del proceso educativo no se transforme en significativo, fracase o no responda a los estudiantes como centro del proceso enseñanza-aprendizaje (Sureda-García \& Gonzáles-Gómez, 2017).

¿Qué factores pueden incidir para que este traslado presencial a la virtualidad se torne en fracaso? ¿Acaso la ausencia de acceso a la tecnología por parte de los estudiantes se convierte en un factor preponderante? ¿Son las estrategias metodológicas empleadas por el docente las inadecuadas para que el aprendizaje del estudiante no sea significativo en esta virtualidad? ¿Cuál es el papel del entorno familiar del estudiante en el proceso de aprendizaje?

Contestar estar preguntas requiere un análisis profundo de la problemática detectada: Los desafíos de la docencia en tiempos de Covid-19. Pero antes de ello, es importante destacar algunos aportes de autores de varias latitudes del mundo de como la educación virtual ha podido inscribirse como alternativa para al menos paliar en alguna medida los impactos que sufre la educación en tiempos de pandemia, por lo tanto, "es importante destacar que la irrupción de las TIC en los contextos escolares pone en evidencia lo 
importante de una nueva definición de roles, especialmente, para los estudiantes y educadores" (Sandoval, 2020).

Por otro lado, se destaca que todos los cambios que la educación debe enfrentar en la virtualidad debe apuntalar a buscar y mejorar la calidad educativa, entre los cuales se hace mención de "la Implementación de recursos tecnológicos, capacitación del personal docente, adaptación de los estudiantes a esta forma de educación, sin olvidar que cada uno de estos aspectos deben enfocarse en buscar la calidad educativa" (Cóndor, 2020). Se observa entonces que todos los aspectos que se puedan incorporar al proceso educativo como tecnologías de información, dispositivos y herramientas tecnológicas, nuevos roles en el docente y estudiante, organización y planificación adaptadas a la realidad virtual, deben buscar como fin primario la calidad educativa, por lo que en tiempos de COVID-19, se hace cuesta arriba desarrollar efectivamente el aprendizaje en los estudiantes, sobre todo, cuando se trata del pensamiento crítico, reflexivo, lógico matemático, por lo desventajoso que ha sido asumir la educación virtual en contexto por pandemia tal como lo indican (González, 2020; Moreno-Correa, 2020). Definitivamente, las estrategias pedagógicas en la virtualidad se convierten en aspectos fundamentales para que el proceso de enseñanza-aprendizaje pueda convertirse en el espacio ideal para la construcción del conocimiento, para los aprendizajes colaborativos (ArgandoñaMendoza et al. 2020).

\section{METODOLOGÍA}

Para el estudio, se trabajó con métodos de investigación cuantitativa, que permitió medir, estimar, y cotejar muestras por medio de la recopilación de datos a través de un procedimiento metodológico descriptivo con diseño no experimental. Para la recolección de la información, se utilizó un cuestionario diseñado con 43 preguntas para obtener respuestas que sean significativas para el estudio. El universo de estudio, fueron 15 docentes y 147 estudiantes del bachillerato de la comunidad educativa Ciudad de Cuenca. Se realizó un muestreo probabilístico estratificado, que implicó dividir la muestra 
en estratos en función de su año de estudio que forma parte de la población $(\mathrm{N})$ del Universo. La investigación se realizó con un 95 por ciento de confiabilidad y un margen de error del \pm 5 por ciento.

Dentro del análisis de datos se definió en función de las características a estudiar en la presente investigación como edad, género, año de estudio, los instrumentos tecnológicos para estudiar, el ambiente donde se encuentra para el proceso de aprendizaje, la composición del hogar, síntomas de violencia intra familiar, emociones, sentimientos, entre otros. Para el cálculo de la muestra de la población estudiantil, se empleó la fórmula de la población finita, que dio como resultado 107 estudiantes a los que se les aplicó el instrumento de recolección de datos. Aplicando los valores a la fórmula de la población finita, se obtuvo el siguiente resultado de 107. Se ha pedido a los participantes que respondan en la escala Likert de cinco puntos que va de 1 = Ningún día, totalmente en desacuerdo, a 5 = Todos los días, Totalmente de acuerdo. Un ítem de muestra es, por ejemplo: "El profesor te anima a estudiar".

Por otro lado, realizado el análisis de fiabilidad arrojó como resulto 0,773 en el indicador Alfa de Cronbach, con 21 preguntas del instrumento de recoleccion de datos utilizado en la investigación. De igual forma, realizado el análisis de fiabilidad aplicada al cuestionario para docentes, arrojó como resultado 0,748 en el indicador Alfa de Cronbach, con 19 preguntas del instrumento aplicado.

\section{RESULTADOS}

De acuerdo a los resultados de la prueba de normalidad se determinó que las todas las preguntas del instrumento de recolección de datos aplicados a estudiantes, fueron de naturaleza paramétrica. La Tabla 1, muestra el comportamiento de las variables. 
Revista Arbitrada Interdisciplinaria KOINONIA

Año VI. Vol VI. N³. Edición Especial: Educación II. 2021

Hecho el depósito de Ley: FA2016000010 ISSN: 2542-3088

FUNDACIÓN KOINONIA (F.K). Santa Ana de Coro. Venezuela.

Tabla 1.

Análisis de normalidad de instrumento de recolección de datos de estudiantes.

\begin{tabular}{lcccc}
\hline & Kolmogorov-Smirnov & & & \\
& Estadístico & gl & Sig. & Observación \\
\hline Género & 0,346 & 102 & 0,000 & Paramétrica \\
Computadora de escritorio (desktop) & 0,328 & 102 & 0,000 & Paramétrica \\
Computadora portátil (laptop) & 0,225 & 102 & 0,000 & Paramétrica \\
Señal de internet & 0,461 & 102 & 0,000 & Paramétrica \\
El lugar donde estudias permite concentrarte & 0,267 & 102 & 0,000 & Paramétrica \\
Relación intrafamiliar durante la pandemia & 0,195 & 102 & 0,000 & Paramétrica \\
Preparación académica durante la pandemia & 0,402 & 102 & 0,000 & Paramétrica \\
Te ha animado a estudiar & 0,254 & 102 & 0,000 & Paramétrica \\
Ha fijado actividades nuevas que puedas hacer & 0,243 & 102 & 0,000 & Paramétrica \\
todos los días & 0,201 & 102 & 0,000 & Paramétrica \\
Te dice que haces las cosas bien & 0,182 & 102 & 0,000 & Paramétrica \\
Te anima a hacer mejor las cosas & 0,244 & 102 & 0,000 & Paramétrica \\
Las actividades en clase y los trabajos que realizo en & & & & \\
clase me ayudan a estudiar & 0,238 & 102 & 0,000 & Paramétrica \\
Me distraigo en clase haciendo garabatos o haciendo & & & & \\
otras cosas & 0,174 & 102 & 0,000 & Paramétrica \\
Habitualmente participo en las discusiones o & & & & \\
actividades que se realizan en la clase virtual & 0,193 & 102 & 0,000 & Paramétrica \\
Pongo gran atención a lo que dice el/la profesor/a & 0,179 & 102 & 0,000 & Paramétrica \\
durante las clases virtuales & 0,180 & 102 & 0,000 & Paramétrica \\
Entiendo todo lo que me enseñan & 0,244 & 102 & 0,000 & Paramétrica \\
Te has enojado con facilidad & 0,159 & 102 & 0,000 & Paramétrica \\
Te has sentido alegre & 0,269 & 102 & 0,000 & Paramétrica \\
Te has sentido nervioso/a & 0,390 & 102 & 0,000 & Paramétrica \\
Te has sentido solo/a & & & &
\end{tabular}

Fuente: Cuestionario. 
De igual forma, aplicada la prubea de normalidad del instrumento de recolección de datos aplicados a docentes, se ilustra en la tabla 2.

\section{Tabla 2.}

Análisis de normalidad de instrumento de recolección de datos de docentes.

\begin{tabular}{|c|c|c|c|c|}
\hline \multicolumn{5}{|c|}{ Shapiro-Wilk } \\
\hline Género & 0,646 & 13 & 0,000 & Paramétrica \\
\hline Alternativa que identifica las clases virtuales & 0,654 & 13 & 0,000 & Paramétrica \\
\hline $\begin{array}{l}\text { Tiempo para planificar clases virtuales, realizar } \\
\text { y corregir tareas. }\end{array}$ & 0,628 & 13 & 0,000 & Paramétrica \\
\hline WhatsApp & 0,619 & 13 & 0,000 & Paramétrica \\
\hline $\begin{array}{l}\text { Redes sociales tipo Facebook, TikTok, } \\
\text { Instagram }\end{array}$ & 0,857 & 13 & 0,036 & \\
\hline $\begin{array}{l}\text { Correo electrónico, videoconferencia vía } \\
\text { Zoom, Meet u otros }\end{array}$ & 0,446 & 13 & 0,000 & Paramétrica \\
\hline Sitio web de la institución & 0,827 & 13 & 0,014 & \\
\hline Blog personal & 0,851 & 13 & 0,029 & \\
\hline Plataforma Teams & 0,706 & 13 & 0,001 & \\
\hline No me puedo comunicar con mis estudiantes & 0,829 & 13 & 0,015 & \\
\hline Otras plataformas educacionales & 0,763 & 13 & 0,003 & \\
\hline $\begin{array}{l}\text { Recurso educativo más utilizado en la } \\
\text { pandemia }\end{array}$ & 0,894 & 13 & 0,110 & \\
\hline Me contacto con mis estudiantes & 0,638 & 13 & 0,000 & Paramétrica \\
\hline Me contacto con los representantes & 0,837 & 13 & 0,020 & \\
\hline $\begin{array}{l}\text { Me contacto con mis compañeros para análisis } \\
\text { educativo }\end{array}$ & 0,720 & 13 & 0,001 & \\
\hline Me contacto con las autoridades superiores & 0,900 & 13 & 0,132 & \\
\hline $\begin{array}{l}\text { Apoyo escolar en casa por parte de padres o } \\
\text { representantes }\end{array}$ & 0,446 & 13 & 0,000 & Paramétrica \\
\hline Capacitación próxima por parte del docente & 0,942 & 13 & 0,487 & \\
\hline $\begin{array}{l}\text { Responsables de educar a los estudiantes en } \\
\text { tecnologías }\end{array}$ & 0,733 & 13 & 0,001 & \\
\hline
\end{tabular}

Fuente: Cuestionario. 
Revista Arbitrada Interdisciplinaria KOINONIA

Año VI. Vol VI. N³. Edición Especial: Educación II. 2021

Hecho el depósito de Ley: FA2016000010 ISSN: 2542-3088

FUNDACIÓN KOINONIA (F.K). Santa Ana de Coro. Venezuela.

Isabel Fabiola Pauta-Ayabaca; Luis Bolívar Cabrera-Berrezueta

La Tabla 3, presenta los valores para la muestra obtenida del universo de estudio en función de los datos demográficos.

Tabla 3.

Datos demográficos de los estudiantes.

\begin{tabular}{|c|c|c|c|}
\hline \multicolumn{4}{|c|}{ Género } \\
\hline & & Frecuencia & Porcentaje \\
\hline \multirow[t]{4}{*}{ Válidos } & Otros & 1 & 0,9 \\
\hline & Femenino & 50 & 47,2 \\
\hline & Masculino & 55 & 51,9 \\
\hline & Total & 106 & 100,0 \\
\hline \multicolumn{4}{|c|}{ Edad } \\
\hline & & Frecuencia & Porcentaje \\
\hline Más de & & 2 & 1,9 \\
\hline 17 a 18 & & 53 & 50,0 \\
\hline 15 a 16 & & 51 & 48,1 \\
\hline Total & & 106 & 100,0 \\
\hline \multicolumn{4}{|c|}{ Año de bachillerato } \\
\hline & & Frecuencia & Porcentaje \\
\hline \multicolumn{2}{|c|}{ Tercero de bachillerato } & 30 & 28,6 \\
\hline \multicolumn{2}{|c|}{ Segundo de bachillerato } & 35 & 33,3 \\
\hline \multicolumn{2}{|c|}{ Primero de bachillerato } & 39 & 37,1 \\
\hline \multicolumn{2}{|l|}{0} & 1 & 1,0 \\
\hline \multicolumn{2}{|c|}{ Total } & 105 & 100,0 \\
\hline \multicolumn{2}{|c|}{ Perdidos Sistema } & 1 & \\
\hline \multicolumn{2}{|c|}{ Total } & 106 & \\
\hline
\end{tabular}

Fuente: Cuestionario. 
Por otro lado, la Tabla 4 ilustra los datos demográficos del instrumento de recolección de datos aplicado a los docentes.

\section{Tabla 4}

Datos demográficos de los docentes.

\begin{tabular}{llcc}
\hline & & Género & \\
\hline \multirow{3}{*}{ Válidos } & Mujer & Frecuencia & Porcentaje \\
& Hombre & 7 & 53,8 \\
Total & Total & 6 & 46,2 \\
& & 13 & 100,0 \\
\hline \multirow{2}{*}{ Válidos } & & 17 & \\
& & Edad & Porcentaje \\
& 56 a 60 años & Frecuencia & 23,1 \\
& 51 a 55 años & 3 & 7,7 \\
& 46 a 50 años & 1 & 7,7 \\
& 41 a 45 años & 1 & 15,4 \\
Perdidos & 36 a 40 años & 2 & 7,7 \\
Total & 31 a 35 años & 1 & 23,1 \\
& 23 a 30 años & 3 & 15,4 \\
\hline & Total & 2 & 100,0 \\
& Sistema & 13 & \\
\hline
\end{tabular}

Fuente: Cuestionario.

\section{Análisis de la infraestructura tecnológica de los estudiantes}

La tabla 5, ilustra la disponibilidad de infrestructura tecnólogica básica de los estudiantes de bachillerato para recibir clases virtuales durante la pandemia que permite determinar el número de casos de estudiantes del Colegio Ciudad de Cuenca que no cuentan con las condiciones necesarias para el aprendizaje de los contenidos curriculares emitidos por los docentes.

De acuerdo al estudio, el 56,2\% de estudiantes no dispone de un computador personal, asi como el $37,1 \%$ no dispone de un computador portátil. Aplicando la media aritmética 
se puede determinar que el $46,65 \%$ de los estudiantes no posee un computador para utilizar en las clases virtuales y realizar las tareas escoloares. Así mismo, el 4,8\% tampoco dispone de alguna conexión a internet a traves de un computador, una portatil, un teléfono celular o una tablet.

Estos datos se convirtien en un factor importante en la dismunución del rendimiento académico de los estudiantes y que puede además traducirse en deserción escolar.

Tabla 5.

Disponibilidad de infraestructura tecnológico para acceso a clases virtuales.

\begin{tabular}{llcccccc}
\hline & \multicolumn{2}{c}{$\begin{array}{c}\text { Computador PC } \\
\text { (escritorio) }\end{array}$} & \multicolumn{2}{c}{ Computador Laptop } & \multicolumn{2}{c}{ Conexión a Internet } \\
\cline { 3 - 8 } & & Frecuencia & Porcentaje & Frecuencia & Porcentaje & Frecuencia & Porcentaje \\
\hline Válidos & 3 o más & 5 & 4,8 & 2 & 1,9 & 3 & 2,9 \\
& Dos & 5 & 4,8 & 13 & 12,4 & 1 & 1,0 \\
& Uno & 30 & 28,6 & 42 & 40,0 & 88 & 83,8 \\
& Ninguno & 59 & 56,2 & 39 & 37,1 & 5 & 4,8 \\
& 0 & 6 & 5,7 & 9 & 8,6 & 8 & 7,6 \\
& Total & 105 & 100,0 & 105 & 100,0 & 105 & 100,0 \\
Perdidos & Sistema & 1 & & 1 & & 1 & \\
Total & & 106 & & 106 & & 106 & \\
\hline
\end{tabular}

Fuente: Cuestionario.

\section{Análisis de las condiciones de estudio y relaciones intrafamiliares}

Por otro lado, un $25,8 \%$ de los estudiantes no dispone de un ambiente adecuado para recibir las clases durante la pandemia que no les permite concentrarse, este porcentaje es ligeramente superior en estudiantes de género masculino. Así mismo, sobre las relaciones intrafamiliares durante la pandemia, el 16,2\% de los estudiantes consultados, mantuvieron una relación difícil y muy difícil con sus familiares. Al relacionar estas dos variables se pudo determinar que el lugar donde el investigado utiliza para estudiar y las relaciones intrafamiliares no están asociadas, pues el coeficiente de Pearson en la prueba Chi-cuadrado es de 0,148 , valor mayor a 0,05 que asume H0 hipótesis nula, por tanto, no asociadas. La tabla 6, muestra el comportamiento de las dos variables. 


\section{Tabla 7.}

Motivación del docente a estudiantes durante clases virtuales en pandemia.

\begin{tabular}{lcccc}
\hline & $\begin{array}{c}\text { Te ha } \\
\text { animado a } \\
\text { estudiar }\end{array}$ & $\begin{array}{c}\text { Ha fijado actividades } \\
\text { nuevas que puedas } \\
\text { hacer todos los días }\end{array}$ & $\begin{array}{c}\text { Te dice que } \\
\text { haces las cosas } \\
\text { bien }\end{array}$ & $\begin{array}{c}\text { Te anima a } \\
\text { hacer mejor } \\
\text { las cosas }\end{array}$ \\
\cline { 2 - 5 } & Porcentaje & Porcentaje & Porcentaje & Porcentaje \\
\hline Todos los días de clase & 35,2 & 23,8 & 15,2 & 28,6 \\
La mayoría de los días de & 35,2 & 34,3 & 19,0 & 20,0 \\
clase & 17,1 & 19,0 & 30,5 & 21,9 \\
Algunos días de clase & 6,7 & 8,6 & 12,4 & 9,5 \\
Pocos días de clase & 5,7 & 3,8 & 8,6 & 6,7 \\
Ningún día de clase & 100,0 & 100,0 & 100,0 & 100,0 \\
Total & 0,9 & 0,9 & 0,9 & 0,9 \\
Perdidos Sistema & & & & \\
\hline
\end{tabular}

Fuente: Cuestionario.

Durante las clases virtuales, el estudiante consultado realizó otras actividades que no están relacionadas con la actividad de aprendizaje. La Taba 6 ilustra el comportamiento de los estudiantes durante clases virtuales en la pandemia, destacándose que el 22,6\% se aburrió y quiso que se terminen las sesiones de clase; el $4 \%$ se quedó dormido y el $11,8 \%$ se distrajo con otras actividades.

Tabla 8.

Actividades que realiza el estudiante durante las clases virtuales en pandemia.

\begin{tabular}{llccc}
\hline Válidos & $\begin{array}{c}\text { Me aburro y quiero } \\
\text { que se acabe }\end{array}$ & $\begin{array}{c}\text { Suelo quedarme } \\
\text { dormido }\end{array}$ & $\begin{array}{c}\text { Me distraigo en clase } \\
\text { haciendo garabatos o } \\
\text { haciendo otras cosas }\end{array}$ \\
\hline & Todos los días & Porcentaje & Porcentaje & Porcentaje \\
& Casi todos los días & 10,8 & 2,0 & 6,9 \\
& Algunos días & 11,8 & 2,0 & 4,9 \\
& Pocos días & 41,2 & 8,8 & 16,7 \\
& Ningún día & 25,5 & 14,7 & 38,2 \\
Perdidos & Total & 10,8 & 66,7 & 26,5 \\
& Sistema & 100,0 & & \\
\hline
\end{tabular}

Fuente: Cuestionario. 
Por otro lado, el $8,8 \%$ de los estudiantes no participan en actividades realizadas en las clases vitales, mientras que el 34,3\% lo hace esporádicamente; así mismo, el 12,7\% no presta atención al docente durante las sesiones.

\section{Tabla 9.}

Participación y atención de los estudiantes en las sesiones de clases.

\begin{tabular}{llcc}
\hline & & $\begin{array}{c}\text { Habitualmente participo en las } \\
\text { discusiones o actividades que se } \\
\text { realizan en la clase virtual }\end{array}$ & $\begin{array}{c}\text { Pongo gran atención a lo que } \\
\text { dice el/la profesor/a durante } \\
\text { las clases virtuales }\end{array}$ \\
\hline Válidos & Porcentaje & Porcentaje \\
& Todos los días & 6,9 & 25,5 \\
& Casi todos los días & 12,7 & 28,4 \\
& Algunos días & 30,4 & 26,5 \\
& Pocos días & 34,3 & 12,7 \\
& Ningún día & 8,8 & 6,9 \\
& O & 6,9 & 100,0 \\
\hline
\end{tabular}

Fuente: Cuestionario.

\section{Análisis de estrategias y recursos didácticos utilizados por los docentes}

Sobre las estrategias que el docente utiliza en clases durante la pandemia, el 7,7\% de docentes traspasan los contenidos planificados para clases presenciales a un formato de clases virtuales; así mismo, el 15,4\% de los docentes diseñan el material de clase utilizando estrategias propias de la virtualidad. Por otro lado, los recursos utilizados por los docentes en su gran mayoría son aquellos depositados en los sitios educativos del Ministerio de Educación, representados por un 29,4\% de docentes consultados. Finalmente, un dato no menor, identifica que el $17,6 \%$ y el $35,3 \%$ no han tenido $u$ ocasionalmente han tenido contacto directo con el estudiante durante las clases virtuales en la pandemia. 
Tabla 10.

Acciones que el docente realiza habitualmente durante clases en pandemia.

\begin{tabular}{llcc}
\hline & & Frecuencia & Porcentaje \\
\hline Válidos & Hago video-clases por Zoom, Google Meet & 9 & 69,2 \\
& Traspaso los contenidos planificados a formato digital & 1 & 7,7 \\
& Diseño las clases nuevas para un formato online & 2 & 15,4 \\
& Adapto el material de clases que tenía planificado usar & 1 & 7,7 \\
& Total & 13 & 100,0 \\
Perdidos & Sistema & 4 & \\
Total & & 17 & \\
\hline
\end{tabular}

Fuente: Cuestionario.

\section{PROPUESTA}

El mundo y particularmnte el país cambió a raíz de la pandemica ocasionada por el CoronaVirus, definitivamente la brecha entre ricos y pobres creció, la desigualdad social se acrecentó, el número de oportunidades para los ciudadanos se limitaron obstenciblemente, ocasionando un sinnúmero de problemas sociales derivados de la falta de oportunidades, de trabajo, de salud y de educación.

Con este panorama, la educación recobra aun más el valor que las sociedades le daban. Se vuelve el escenario ideal para preparar mas que profesionales, a ciudadanos que solidariamente puedan propiciar soluciones a las multiples problemáticas que el ciudadano vive diariamente; por tanto, la educacion, debe convertirse en el eje motor del verdadero cambio que las sociedades necesitan para vivir armónicamente.

Entonces, siendo los docentes parte fundamental del sistema educativo, ¿qué desafios tienen en este nuevo escenario mundial?

A simple vista, deben convertirse en misioneros de la transformacion social, en artifices de formar ciudadanos con un nuevo modelo mental, académico y social; deben convertirse en agricultores de una nueva tierra, donde su siembra permita cosechar hombres y mujeres que solidariamente busquen el bien comun. Exige por tanto, extremar no sólo estrategias pedagógicas acordes a la virtualidad, sino exige redoblar el 
compromiso y la vocación para lograr desde su accionar, la reducción significativa del fracaso escolar y la deserción en esta época.

Debe ingeniarse nuevas formas de captar la atención del estudiante, debe buscar el aprendizaje colaborativo, buscar estimular la síntesis de la información al que accede el estudiante, debe redefinir su rol en la virtualidad, convertirse en guía y no en un simple espectador de la enseñanza. Para ello, se propone una metodología que permita al docente enfrentar los desafíos en estas circunstancias.

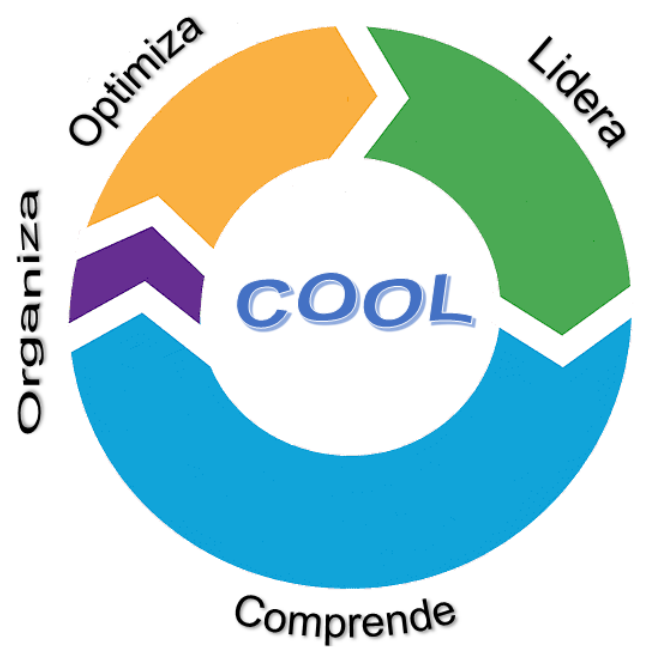

Figura 1. Propuesta de metodología COOL.

Elaboración: Los autores.

Comprende. - Consiste en conocer de cerca la realidad del estudiante; familiarizarse con sus virtudes y defectos, con sus sueños, sus ilusiones, sus miedos. Para ello, el docente debe acercarse sigilosamente, observar cuidadosamente los movimientos del estudiante, utilizar instrumentos de diagnóstico que le den información valiosa para saber con quién se enfrenta, que realidad hay detrás. Debe, por tanto: Investigar, reunir, escuchar, analizar, entender, la realidad del estudiante. 
Organiza. - Es necesario sintetizar la información obtenida, esquematízalo a través de mapas mentales; también se puede organizar una ficha individual del estudiante cruzando variables obtenidas en el paso anterior. Finalmente empodera al estudiante sobre la visión de él ante el mundo, y la visión del mundo ante él.

Estas dos etapas son importantes a la hora de redireccionar el contenido curricular de la asignatura, las dos primeras semanas de clase son el espacio propicio para logar el objetivo planteado.

Optimiza. - Los recursos tanto humanos, técnicos, tecnológicos, metodológicos son fundamentales, pero aún más el tiempo. Organiza las clases en función del diagnóstico preliminar, los contenidos curriculares son importantes, pero más importante es cómo se puede transmitir al estudiante, no como una mera repetición de conceptos y definiciones que bien el estudiante puede encontrarlo en la web, sino de tu propia experiencia y de la visión que tenga el estudiante.

Lidera. - Con el ejemplo, con la experiencia, ilustra como el aprendizaje colaborativo puede incidir fuertemente en el estudiante. Agrupa el más fuerte con el más débil, el artístico con el físico, el soñador con el iluso, el cauto con el apresurado. Da tareas de interés, que motiven al grupo a buscar, a analizar, a encontrar una "verdad". Es necesario armar redes de conocimiento, el conectivismo es una buena opción para ello.

Los desafíos son grandes, el proceso de aprendizaje-aprendizaje-enseñanza, debe propiciar la seguridad, la confianza, la estabilidad emocional, la certeza de un acompañamiento continuo, debe fortalecer los lazos familiares, los valores como punto de partida y como fin mismo, para el crecimiento personal y profesional del estudiante. 


\section{CONCLUSIONES}

Desde la visión de los estudiantes, el estudio revela que el docente debe buscar alternativas pedagógicas diferentes a las utilizadas para lograr captar la atención del estudiante en las sesiones de clases virtuales. Incursionar en herramientas de Gamificación puede convertirse en una alternativa válida.

El estudio concluye, además, que no existe evidencia suficiente que el espacio o lugar donde el estudiante recibe sus clases virtuales y la relación que mantiene al interior de su familia inciden directamente en el rendimiento académico, para que este se convierta en fracaso o deserción. Sin embargo, puede convertirse en un factor detonante, si el docente y las autoridades académicas de la institución no detectan a tiempo este factor. En este sentido, es necesario mencionar que, debido al confinamiento prolongado en la ciudad y el país, los estudiantes ya están sufriendo algún grado de angustia psicológica, miedo, depresión, inseguridad, amenaza, entre otros. Por lo tanto, los docentes y la institución en sí misma, debe asumir estos desafíos para que los estudiantes puedan aprender significativamente.

Debe entonces, en medio de esta crisis sanitaria mundial, la salud mental y el bienestar de los estudiantes convertirse en una prioridad para los docentes. Debe prestar más atención a la satisfacción de las necesidades psicológicas básicas de los estudiantes para que no solo su experiencia de aprendizaje virtual sea positiva y gratificante, sino que también se convierta en un instrumento para que los estudiantes resistan mejor a otros desafíos similares en el futuro.

Los docentes enfrentan desafíos que exigen de él, una mayor preparación, un profundo acercamiento al estudiante, una estrecha relación con el entorno familiar; exige, simplemente vocación, pasión y amor por lo que hace. 


\section{FINANCIAMIENTO}

No monetario.

\section{AGRADECIMIENTO}

A la Jefatura de Posgrados de la Universidad Católica de Cuenca por permitir el desarrollo y fomento de la investigación.

\section{REFERENCIAS CONSULTADAS}

Argandoña-Mendoza, M., García-Mejía, R., Ayón-Parrales, E., \& Zambrano-Zambrano, Y. (2020). Investigación e innovación educativa: Reto escolar por COVID-19 en el Ecuador [Educational research and innovation: School challenge due to COVID19 in Ecuador]. EPISTEME KOINONIA, 3(5), 162-182. http://dx.doi.org/10.35381/e.k.v3i5.726

Cóndor, O. (2020). Educar en tiempos de COVID-19 [Educating in times of pandemic]. CienciAmérica, 9(2), 31. https://doi.org/10.33210/ca.v9i2.281

García-Cedeño, G., Vélez-Loor, M., Franco-Zambrano, C., \& Ormaza-Bermello, M. (2020). Educación por competencias: Una posibilidad reordenadora del currículo pensada en emergencia escolar por COVID-19 [Education by competencies: A possibility to rearrange the curriculum thought about a school emergency due to COVID-19]. EPISTEME KOINONIA, $3(5)$ 223-237. http://dx.doi.org/10.35381/e.k.v3i5.770

González, R. (2020). El reto de enseñar y aprender interpretación signada en tiempos de coronavirus [The challenge of teaching and learning interpretation signed in times of coronavirus]. Revista de Estilos de Aprendizaje, 13(Especial), 17-31

Hurtado-Talavera, F. (2020). La Educación En Tiempos De Pandemia: Los Desafios De La Escuela Del Siglo Xxi [Education in Pandemic Time. Challenges of 21st Century School]. Revista Arbitrada Del Centro De Investigación Y Estudios Gerenciales, 176-187. https://n9.cl/3bau6

Moreno, S. (2020). La innovación educativa en los tiempos del Coronavirus[ Educational innovation in times of Coronavirus]. Salutem Scientia Spiritus, 6(1), 14-26. 
Quevedo-Álava, R., Corrales-Moreno, L., Palma-Delgado, G., \& Mendoza-Suárez, G. (2020). Psicopedagogía y TIC en período de COVID-19. Una reflexión para el aprendizaje significativo [Psychopedagogy and ICT in the COVID-19 period. A reflection for meaningful learning]. EPISTEME KOINONIA, 3(5), 202-222. http://dx.doi.org/10.35381/e.k.v3i5.769

Rodríguez-Tablado, S., García-Herrera, D., Cabrera-Berrezueta, L., \& Erazo-Álvarez, J. (2020). Tecnologías aplicadas en el proceso enseñanza-aprendizaje durante el COVID-19: Un análisis en Educación Básica [Technologies applied in the teachinglearning process during COVID-19: An analysis in Basic Education]. EPISTEME KOINONIA, 3(6), 4-31. http://dx.doi.org/10.35381/e.k.v3i6.813

Sandoval, C. H. (2020). La Educación en Tiempo del Covid-19 Herramientas TIC: El Nuevo Rol Docente en el Fortalecimiento del Proceso Enseñanza Aprendizaje de las Prácticas Educativa Innovadoras[The new teaching role in the teachinglearning process of innovatived practices]. Revista Tecnológica-Educativa Docentes 2.0, 9(2), 24-31. https://doi.org/10.37843/rted.v9i2.138

Sureda-García, B., \& Gonzáles-Gómez, S. (2017). História da educación [History of education]. Revista História Da Educação, 21(51), 229-251.

(C2021 por los autores. Este artículo es de acceso abierto y distribuido según los términos y condiciones de la licencia Creative Commons Atribución-NoComercial-Compartirlgual 4.0 Internacional (CC BY-NC-SA 4.0) (https://creativecommons.org/licenses/by-nc-sa/4.0/). 